\title{
SOME INDUCED AGGREGATING OPERATORS WITH FUZZY NUMBER INTUITIONISTIC FUZZY INFORMATION AND THEIR APPLICATIONS TO GROUP DECISION MAKING
}

\author{
GUIWU WEI*, XIAOFEI ZHAO, RUI LIN \\ Department of Economics and Management, Chongqing University of Arts and Sciences \\ Yongchuan 402160, China \\ *Corresponding author,E-mail:weiguiwu@ 163.com
}

Received: 22-12-2008

Accepted: 27-11-2009

\begin{abstract}
With respect to multiple attribute group decision making (MAGDM) problems in which both the attribute weights and the expert weights take the form of real numbers, attribute values take the form of fuzzy number intuitionistic fuzzy numbers, a new group decision making analysis method is developed. Firstly, some operational laws of fuzzy number intuitionistic fuzzy numbers, score function and accuracy function of fuzzy number intuitionistic fuzzy numbers are introduced. Then a new aggregation operator called induced fuzzy number intuitionistic fuzzy ordered weighted geometric (I-FIFOWG) operator is proposed, and some desirable properties of the I-FIFOWG operators are studied, such as commutativity, idempotency and monotonicity. An I-FIFOWG and FIFWG (fuzzy number intuitionistic fuzzy weighted geometric) operators-based approach is developed to solve the MAGDM under the fuzzy number intuitionistic fuzzy environment. Furthermore, we propose the induced fuzzy number intuitionistic fuzzy ordered weighted averaging (I-FIFOWA) operator. Finally, an illustrative example is given to verify the developed approach and to demonstrate its practicality and effectiveness. Finally, an illustrative example is given to verify the developed approach.
\end{abstract}

Keywords: Fuzzy number intuitionistic fuzzy numbers; operational laws; fuzzy number intuitionistic fuzzy weighted geometric (FIFWG) operator; induced fuzzy number intuitionistic fuzzy ordered weighted geometric (IFIFOWG) operator

\section{Introduction}

Atanassov [1,2] introduced the concept of intuitionistic fuzzy set (IFS), which is a generalization of the concept of fuzzy set [3]. The intuitionistic fuzzy set has received more and more attention since its appearance[4-17, 2428, 33-36]. Gau and Buehrer [4] introduced the concept of vague set. But Bustince and Burillo [5] showed that vague sets are intuitionistic fuzzy sets. $\mathrm{Li}$ [6] investigated multiple attribute decision making with intuitionistic fuzzy information and constructed several linear programming models to generate optimal weights for attribute. Lin [7] presented a new method for handling multiple attribute fuzzy decision making problems, where the characteristics of the alternatives are represented by intuitionistic fuzzy sets. The proposed method allows the degrees of satisfiability and non-satisfiability of each alternative with respect to a set of attribute to be represented by intuitionistic fuzzy sets, respectively. Furthermore, the proposed method allows the decision-maker to assign the degree of membership and the degree of non-membership of the attribute to the fuzzy concept "importance." Xu [8] developed some geometric aggregation operators, such as the intuitionistic fuzzy weighted geometric (IFWG) operator, the intuitionistic fuzzy ordered weighted geometric (IFOWG) operator, and the intuitionistic fuzzy hybrid geometric (IFHG) operator and gave an application of the IFHG operator to multiple attribute group decision making with intuitionistic fuzzy information. $\mathrm{Xu}$ [9] developed some arithmetic 
aggregation operators, such as the intuitionistic fuzzy arithmetic averaging (IFAA) operator and the intuitionistic fuzzy weighted averaging (IFWA) operator. $\mathrm{Xu}[10]$ further developed the intuitionistic fuzzy ordered weighted averaging (IFOWA) operator, and the intuitionistic fuzzy hybrid aggregation (IFHA) operator. Later, Atanassov and Gargov [11-12] further introduced the interval-valued intuitionistic fuzzy set (IVIFS), which is a generalization of the IFS. The fundamental characteristic of the IVIFS is that the values of its membership function and non-membership function are intervals rather than exact numbers. Wang[13] used evidential reasoning algorithms to solve multiple attribute decision making in which the information on the attribute's weights is incomplete certain and attribute's values is interval intuitionistic fuzzy numbers. $\mathrm{Xu}$ [14] developed some geometric aggregation operators, such as the interval-valued intuitionistic fuzzy weighted geometric (IIFWG) operator and the interval-valued intuitionistic fuzzy geometric (IIFG) operator and gave an application of the IIFWG and IIFG operators to multiple attribute group decision making with interval-valued intuitionistic fuzzy information. $\mathrm{Xu}$ [15] developed some arithmetic aggregation operators, such as the interval-valued intuitionistic fuzzy weighted averaging (IIFWA) operator, the interval-valued intuitionistic fuzzy ordered weighted averaging (IIFOWA) operator, and the interval-valued intuitionistic fuzzy hybrid aggregation (IIFHA) operator and gave an application of the IIHA operator to multiple attribute group decision making with interval-valued intuitionistic fuzzy information. Liu and Yuan [16] introduced the concept of fuzzy number intuitionistic fuzzy set (FNIFS) which fundamental characteristic of the FNIFS is that the values of its membership function and non-membership function are triangular fuzzy numbers rather than exact numbers. Wang[17] propose some aggregation operators, including fuzzy number intuitionistic fuzzy weighted geometric (FIFWG) operator, fuzzy number intuitionistic fuzzy ordered weighted geometric (FIFOWG) operator and fuzzy number intuitionistic fuzzy hybrid geometric (FIFHG) operator and develop an approach to multiple attribute group decision making (MAGDM) based on the FIFWG and the FIFHG operators with fuzzy number intuitionistic fuzzy information. Wang [24] propose the fuzzy number intuitionistic fuzzy weighted averaging (FIFWA) operator, fuzzy number intuitionistic fuzzy ordered weighted averaging (FIFOWA) operator and fuzzy number intuitionistic fuzzy hybrid aggregation (FIFHA) operator.

With respect to the MAGDM problems, in which both the attribute weights and the expert weights take the form of real numbers, attribute values take the form of fuzzy number intuitionistic fuzzy numbers, "how to aggregate these fuzzy number intuitionistic fuzzy data by using the induced aggregation operators?" is an interesting research topic and is worth paying attention to. In this paper, we shall develop some induced aggregation operators with fuzzy number intuitionistic fuzzy information. To do so, the remainder of this paper is set out as follows. In the next section, we introduce some basic concepts related to fuzzy number intuitionistic fuzzy sets and some operational laws of fuzzy number intuitionistic fuzzy numbers. In Section 3 we shall develop a new operator called induced fuzzy number intuitionistic fuzzy ordered weighted geometric (I-FIFOWG) operator which is an extension of induced ordered weighted geometric (IOWG) operator proposed by Yager and Filev [18] and study some desirable properties of the I-FIFOWG operators, such as commutativity, idempotency and monotonicity. In Section 4, we shall develop a new operator called induced fuzzy number intuitionistic fuzzy ordered weighted averaging (I-FIFOWA) operator which is an extension of induced ordered weighted averaging (IOWA) operator [25] and study some desirable properties of the I-FIFOWA operators, such as commutativity, idempotency and monotonicity. In Section 5, An I-FIFOWG and FIFWG operators-based approach is developed to solve the MAGDM under the fuzzy number intuitionistic fuzzy environment. In Section 6, an illustrative example is pointed out. In Section 7, we conclude the paper and give some remarks.

\section{Preliminaries}

Atanassov [1-2] extended the fuzzy set to the IFS, shown as follows:

Definition 1. An IFS $A$ in $X$ is given by

$$
A=\left\{\left\langle x, \mu_{A}(x), v_{A}(x)\right\rangle \mid x \in X\right\}
$$

Where $\mu_{A}: X \rightarrow[0,1]$ and $v_{A}: X \rightarrow[0,1]$, with the condition

$$
0 \leq \mu_{A}(x)+v_{A}(x) \leq 1, \forall x \in X
$$

The numbers $\mu_{A}(x)$ and $v_{A}(x)$ represent, respectively, the membership degree and nonmembership degree of the element $x$ to the set $A$. And let $\pi_{A}(x)=1-\mu_{A}(x)-v_{A}(x)$, then $\pi_{A}(x)$ is called the degree of indeterminacy of $x$ to $A$ [1-2].

Definition 2. Let $X$ is a universe of discourse, An IVIFS $\AA^{\prime}$ over $X$ is an object having the form [11-12]:

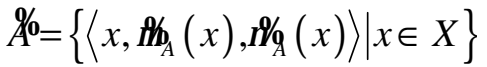


Where $\mu_{A}(x) \subset[0,1]$ and $\% \%(x) \subset[0,1]$ are interval numbers, and

$$
0 \leq \sup (\mu / \rho(x))+\sup (\mathrm{v} \%(x)) \leq 1, \forall x \in X
$$

For convenience, let $\mu_{\AA}(x)=[a, b]$, $\nu_{A}(x)=[c, d]$, so $\AA \stackrel{\varrho}{=}([a, b],[c, d])$.

Liu and Yuan [16] introduced the concept of fuzzy number intuitionistic fuzzy set (FNIFS) which fundamental characteristic of the FNIFS is that the values of its membership function and non-membership function are triangular fuzzy numbers.

Definition 3. Let $X$ is a universe of discourse, An FNIFS $A^{\prime}$ over $X$ is an object having the form:

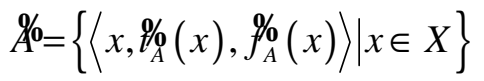

Where $\quad \% / q(x) \subset[0,1]$ and $\quad f_{A}^{\circ}(x) \subset[0,1]$ are triangular fuzzy numbers, and

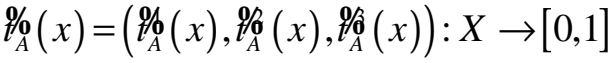

$$
\begin{aligned}
& f_{A}^{q} / q(x)=\left(f_{A}^{\prime / q}(x), f_{A}^{\circ / o}(x), f_{A}^{f o g}(x)\right): X \rightarrow[0,1] \\
& 0 \leq \% \text { \% }(x)+f_{A}^{\circ}(x) \leq 1, \forall x \in X
\end{aligned}
$$

For convenience, let $\quad f_{A} /(x)=(a, b, c)$, $f_{A}^{\circ}(x)=(l, m, p)$, so $\AA$ 으 $\langle(a, b, c),(l, m, p)\rangle$.

Definition 4. Let $\mathscr{Q}_{1} \mathrm{o}=\left\langle\left(a_{1}, b_{1}, c_{1}\right),\left(l_{1}, m_{1}, p_{1}\right)\right\rangle$ and $\mathscr{d}_{2} \mathrm{o}=\left\langle\left(a_{2}, b_{2}, c_{2}\right),\left(l_{2}, m_{2}, p_{2}\right)\right\rangle$ be two fuzzy number intuitionistic fuzzy values, then

(1) $q_{1} \propto \mathscr{Q}_{2} \mathrm{o}=\left\langle\left(a_{1} a_{2}, b_{1} b_{2}, c_{1} c_{2}\right)\right.$,

$$
\left.\left(l_{1}+l_{2}-l_{1} l_{2}, m_{1}+m_{2}-m_{1} m_{2}, p_{1}+p_{2}-p_{1} p_{2}\right)\right\rangle ;
$$

(2) $\mathscr{Q}_{1}^{\lambda} \mathrm{o}=\left\langle\left(a_{1}^{\lambda}, b_{1}^{\lambda}, c_{1}^{\lambda}\right)\right.$,

$$
\left.\left(1-\left(1-l_{1}\right)^{\lambda}, 1-\left(1-m_{1}\right)^{\lambda}, 1-\left(1-p_{1}\right)^{\lambda}\right)\right\rangle, \lambda \geq 0 \text {; }
$$

Definition 5. Let $\mathscr{V} \sigma\langle(a, b, c),(l, m, p)\rangle$ be a fuzzy number intuitionistic fuzzy value, a score function $S$ of a fuzzy number intuitionistic fuzzy value can be represented as follows [17]:

$$
\begin{gathered}
S\left(\partial b=\frac{a+2 b+c}{4}-\frac{l+2 m+p}{4},\right. \\
S(\&) \in[-1,1] .
\end{gathered}
$$

Definition 6. Let $\mathscr{d} \sigma\langle(a, b, c),(l, m, p)\rangle$ be a fuzzy number intuitionistic fuzzy value, an accuracy function $H$ of a fuzzy number intuitionistic fuzzy value can be represented as follows:

$$
\begin{gathered}
H(a)_{0}=\frac{(a+2 b+c)+(l+2 m+p)}{4}, \\
H\left(a b_{b} \in[0,1] .\right.
\end{gathered}
$$

to evaluate the degree of accuracy of the fuzzy number intuitionistic fuzzy value $\mathscr{V} \sigma\langle(a, b, c),(l, m, p)\rangle$, where $H(A) \in[0,1]$. The larger the value of $H(A)_{0}$, the more the degree of accuracy of the fuzzy number intuitionistic fuzzy value $\mathscr{d}$ is.

As presented above, the score function $S$ and the accuracy function $H$ are, respectively, defined as the difference and the sum of the membership function $\mu / \rho(x)$ and the non-membership function $\% \%(x)$. Xu [8] showed that the relation between the score function $S$ and the accuracy function $H$ is similar to the relation between mean and variance in statistics. Based on the score function $S$ and the accuracy function $H$, in the following, we shall give an order relation between two fuzzy number intuitionistic fuzzy values, which is defined as follows:

Definition 7. Let $\mathscr{C} / \mathrm{c}$ and $\mathscr{Q} / \mathrm{o}$ be two fuzzy number intuitionistic fuzzy values, $s(\mathscr{Q} /)$ and $s(\mathscr{Q} /)$ be the scores of $\mathscr{d}$ and $b^{2}$, respectively, and let $H\left(q_{1}\right)$ and $H(d 2)$ be the accuracy degrees of $d$ and $b^{\prime}$, respectively, then if $S(\mathscr{C})<S(\not{b})$, then $\mathscr{Q}$ is smaller than $b^{\circ}$, denoted by $\mathscr{d} \times b^{\circ}$; if $S(\not{b} \phi=S(\not{b})$, then if $H(\mathscr{a})=H(\not{b})$, then $\mathscr{Q}$ and $\mathscr{b}$ represent the same information, denoted by $\mathscr{d} \sigma b^{\prime} ;$ (2) if $H(\not{a})<H(\not{b})$, $\mathscr{d}$ is smaller than $\mathfrak{b}$, denoted by $\mathscr{d}<\vec{b}$.

\section{Induced fuzzy number intuitionistic fuzzy ordered weighted geometric (I-FIFOWG) operator}

Definition 7. Let $\mathscr{Q}_{j}=\left\langle\left(a_{j}, b_{j}, c_{j}\right),\left(l_{j}, m_{j}, p_{j}\right)\right\rangle$ $(j=1,2, \mathrm{~L}, n)$ be a collection of fuzzy number 
intuitionistic fuzzy values, and let FIFWG: $Q^{n} \rightarrow Q$, if

$F I F W G_{\omega}\left(\& \&_{1} Q \&_{2} \mathrm{~L}, \mathscr{Q}_{n}\right)=\prod_{j=1}^{n} q_{j}$

$=\left\langle\left(\prod_{j=1}^{n} a_{j}^{\omega_{j}}, \prod_{j=1}^{n} b_{j}^{\omega_{j}}, \prod_{j=1}^{n} c_{j}^{\omega_{j}}\right)\right.$,

$\left.\left(1-\prod_{j=1}^{n}\left(1-l_{j}\right)^{\omega_{j}}, 1-\prod_{j=1}^{n}\left(1-m_{j}\right)^{\omega_{j}}, 1-\prod_{j=1}^{n}\left(1-p_{j}\right)^{\omega_{j}}\right)\right\rangle$

where $\omega=\left(\omega_{1}, \omega_{2}, \mathrm{~L}, \omega_{n}\right)^{T}$ be the weight vector of $\approx \rho \mathrm{o}(j=1,2, \mathrm{~L}, n)$, and $\omega_{j}>0, \sum_{j=1}^{n} \omega_{j}=1$, then FIFWG is called the fuzzy number intuitionistic fuzzy weighted geometric (FIFWG) operator [17].

Example 1. Assume $\omega=(0.2,0.1,0.3,0.4)$,

$\mathscr{V}_{1}=\langle(0.1,0.2,0.3),(0.5,0.6,0.7)\rangle$,

$\mathscr{q} \mathrm{O}=\langle(0.4,0.5,0.6),(0.3,0.3,0.4)\rangle$,

$\mathscr{C}_{3} \mathrm{O}=\langle(0.4,0.4,0.5),(0.4,0.4,0.5)\rangle$, and

$\mathscr{q} 0=\langle(0.3,0.4,0.5),(0.3,0.4,0.4)\rangle$, then

$\mathrm{FIFWG}_{\omega}\left(d_{1}, q_{2}, d_{3}, d_{4}\right)$

$=\left\langle\left(0.1^{0.2} \times 0.4^{0.1} \times 0.4^{0.3} \times 0.3^{0.4}\right.\right.$,

$0.2^{0.2} \times 0.5^{0.1} \times 0.4^{0.3} \times 0.4^{0.4}$

$\left.0.3^{0.2} \times 0.6^{0.1} \times 0.5^{0.3} \times 0.5^{0.4}\right)$,

$\left(1-(1-0.5)^{0.2} \times(1-0.3)^{0.1} \times(1-0.4)^{0.3} \times(1-0.3)^{0.4}\right.$,

$1-(1-0.6)^{0.2} \times(1-0.3)^{0.1} \times(1-0.4)^{0.3} \times(1-0.4)^{0.4}$,

$\left.1-(1-0.7)^{0.2} \times(1-0.4)^{0.1} \times(1-0.5)^{0.3} \times(1-0.4)^{0.4}\right)$

$=\langle(0.270,0.356,0.460),(0.625,0.562,0.495)\rangle$

Definition 8. Let $\&_{j}=\left\langle\left(a_{j}, b_{j}, c_{j}\right),\left(l_{j}, m_{j}, p_{j}\right)\right\rangle$ $(j=1,2, \mathrm{~L}, n)$ be a collection of fuzzy number intuitionistic fuzzy values, An fuzzy number intuitionistic fuzzy ordered weighted geometric (FIFOWG) operator of dimension $n$ is a mapping FIFOWG: $Q^{n} \rightarrow Q$, that has an associated weight vector $w=\left(w_{1}, w_{2}, \mathrm{~L}, w_{n}\right)^{T}$ such that $w_{j}>0$ and $\sum_{j=1}^{n} w_{j}=1$. Furthermore,

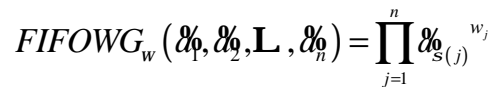

$=\left\langle\left(\prod_{j=1}^{n} a_{\sigma(j)}{ }^{w_{j}}, \prod_{j=1}^{n} b_{\sigma(j)}{ }^{w_{j}}, \prod_{j=1}^{n} c_{\sigma(j)}{ }^{w_{j}}\right)\right.$,

$\left.\left(1-\prod_{j=1}^{n}\left(1-l_{\sigma(j)}\right)^{w_{j}}, 1-\prod_{j=1}^{n}\left(1-m_{\sigma(j)}\right)^{w_{j}}, 1-\prod_{j=1}^{n}\left(1-p_{\sigma(j)}\right)^{w_{j}}\right)\right)$

where $(\sigma(1), \sigma(2), \mathrm{L}, \sigma(n))$ is a permutation of $(1,2, \mathrm{~L}, n)$, such that $\mathscr{O}_{\sigma(j-1)} \geq \mathscr{Q}_{\sigma(j)}$ for all $j=2, \mathrm{~L}, n$. [17].

Example 2. Let $\mathscr{Q} / 0=\langle(0.3,0.4,0.5),(0.2,0.3,0.4)\rangle$, $8 / 2=\langle(0.3,0.3,0.3),(0.4,0.5,0.6)\rangle$ $\mathscr{A} / 0=\langle(0.5,0.5,0.5),(0.3,0.3,0.3)\rangle \quad, \quad$ and $\mathscr{Q}_{4}=\langle(0.1,0.2,0.2),(0.6,0.7,0.8)\rangle \quad$ be four intuitionistic fuzzy values, by (4), we calculate the scores of $\mathscr{V} /(j=1,2,3,4)$ :

$$
\begin{aligned}
& S\left(\&_{1}\right)=0.1, S\left(\&_{2}\right)=-0.2 \\
& S\left(\&_{3}\right)=0.2, S\left(\&_{4}\right)=-0.53
\end{aligned}
$$

Since

$$
S(\& / 3)>S(\& / 0)>S(\& / 2)>S(\& / 0)
$$

thus

$$
\begin{aligned}
& \mathscr{d}_{\sigma(1)}=\langle(0.5,0.5,0.5),(0.3,0.3,0.3)\rangle, \\
& \mathscr{d}_{\sigma(2)}=\langle(0.3,0.4,0.5),(0.2,0.3,0.4)\rangle, \\
& \mathscr{\sigma}_{\sigma(3)}=\langle(0.3,0.3,0.3),(0.4,0.5,0.6)\rangle, \\
& \mathscr{q} \sigma(4)_{\sigma}=\langle(0.1,0.2,0.2),(0.6,0.7,0.8)\rangle
\end{aligned}
$$

Suppose that $w=(0.2,0.3,0.4,0.1)$ is the weighting vector of the FIFOWG operator. Then, by (7), it follows that 
FIFOWG $_{w}\left(\&_{1}, \&_{2} 9 \% \&_{3} \&_{4}\right)$

$$
\begin{aligned}
= & \left\langle\left( 0.5^{0.2} \times 0.3^{0.3} \times 0.3^{0.4} \times 0.1^{0.1},\right.\right. \\
& 0.5^{0.2} \times 0.4^{0.3} \times 0.3^{0.4} \times 0.2^{0.1}, \\
& \left.0.5^{0.2} \times 0.5^{0.3} \times 0.3^{0.4} \times 0.2^{0.1}\right), \\
& \left(1-(1-0.3)^{0.2} \times(1-0.2)^{0.3} \times(1-0.4)^{0.4} \times(1-0.6)^{0.1},\right. \\
1- & (1-0.3)^{0.2} \times(1-0.3)^{0.3} \times(1-0.5)^{0.4} \times(1-0.7)^{0.1}, \\
1- & \left.(1-0.3)^{0.2} \times(1-0.4)^{0.3} \times(1-0.6)^{0.4} \times(1-0.8)^{0.1}\right) \\
= & \langle(0.214,0.291,0.297),(0.541,0.451,0.353)\rangle
\end{aligned}
$$

In the following, we shall develop an induced fuzzy number intuitionistic fuzzy ordered weighted geometric (I-FIFOWG) operator.

Definition 9. An induced fuzzy number intuitionistic fuzzy ordered weighted geometric (I-FIFOWG) operator is defined as follows:

$\mathrm{I}-F I F O W G_{\mathrm{w}}\left(\left\langle u_{1}, \& \phi,\left\langle u_{2}, \& \hat{\alpha}, \mathrm{L},\left\langle u_{n}, 8 \hat{q} q\right)\right.\right.\right.$

$$
\begin{aligned}
& =\prod_{j=1}^{n} g_{\sigma(j)} \sigma_{\sigma_{j}}^{w_{j}} \\
& =\left\langle\left(\prod_{j=1}^{n} a_{\sigma(j)}{ }^{w_{j}}, \prod_{j=1}^{n} b_{\sigma(j)}{ }^{w_{j}}, \prod_{j=1}^{n} c_{\sigma(j)}{ }^{w_{j}}\right),\right. \\
& \left.\left(1-\prod_{j=1}^{n}\left(1-l_{\sigma(j)}\right)^{w_{j}}, 1-\prod_{j=1}^{n}\left(1-m_{\sigma(j)}\right)^{w_{j}}, 1-\prod_{j=1}^{n}\left(1-p_{\sigma(j)}\right)^{w_{j}}\right)\right\rangle
\end{aligned}
$$

where $w=\left(w_{1}, w_{2}, \mathrm{~L}, w_{n}\right)^{T}$ is a weighting vector, such that $w_{j} \in[0,1], \sum_{j=1}^{n} w_{j}=1, j=1,2, \mathrm{~L}, n$, $\mathscr{Q}_{\sigma(j)}=\left\langle\left(a_{\sigma(j)}, b_{\sigma(j)}, c_{\sigma(j)}\right),\left(l_{\sigma(j)}, m_{\sigma(j)}, p_{\sigma(j)}\right)\right\rangle$ is the $\mathscr{Q} \mathbb{C}_{i}$ value of the FIFOWG pair $\left\langle u_{i}, \mathscr{Q}_{i} \mathrm{O}\right\rangle$ having the jth largest $u_{i}\left(u_{i} \in[0,1]\right)$, and $u_{i}$ in $\left\langle u_{i}, \mathscr{Q}_{i} \mathrm{Q}\right\rangle$ is referred to as the order inducing variable and $\mathscr{Q} / \mathrm{c}$ as the fuzzy number intuitionistic fuzzy values.

The I-FIFOWG operator has the following properties similar to those of the IOWG operator [18].

Theorem 1 (Commutativity).

$\left.\mathrm{I}^{-F I F O W G}{ }_{w}\left(\left\langle u_{1}, \mathscr{O}_{1} \mathrm{O}\right\rangle,\left\langle u_{2}, \mathscr{Q}_{2} \mathrm{O}\right\rangle, \mathrm{L},\left\langle u_{n}, \mathscr{Q}_{n}\right\rangle\right)\right)$

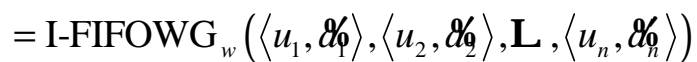

where $\left.\left(\left\langle u_{1}, \mathscr{Q} / 0\right),\left\langle u_{2}, \mathscr{Q} \%\right\rangle, \mathrm{L},\left\langle u_{n}, \mathscr{W}\right\rangle\right\rangle\right) \quad$ is any permutation of $\left(\left\langle u_{1}, \mathscr{Q}_{1} \mathrm{O}\right\rangle,\left\langle u_{2}, \mathscr{Q}_{2} \mathrm{O}\right\rangle, \mathrm{L},\left\langle u_{n}, \mathscr{Q}_{n} \mathrm{O}\right\rangle\right)$.

Proof. Let

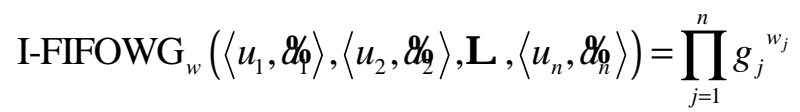

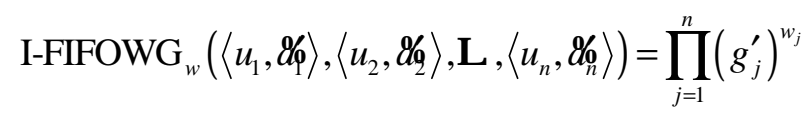

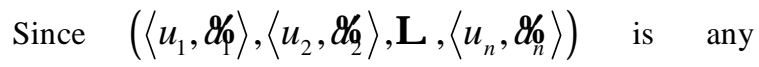
permutation of $\left(\left\langle u_{1}, \mathscr{O}_{1} \mathrm{O},,\left\langle u_{2}, \mathscr{O}_{2} \mathrm{O}\right\rangle, \mathrm{L},\left\langle u_{n}, \mathscr{Q}_{n}\right\rangle\right)\right)$, we have $g_{j}=g_{j}^{\prime}(j=1,2, \mathrm{~L}, n)$, then

$\left.\left.\mathrm{I}^{-F I F O W G}{ }_{w}\left(\left\langle u_{1}, \mathscr{Q}_{1} \mathrm{\rho}\right\rangle,\left\langle u_{2}, \mathscr{Q}_{2}\right\rangle\right\rangle, \mathrm{L},\left\langle u_{n}, \mathscr{Q}_{n}\right\rangle\right\rangle\right)$

$\left.=\mathrm{I}^{-F I F O W G}{ }_{w}\left(\left\langle u_{1}, \mathscr{Q} / \mathrm{\rho}\right\rangle,\left\langle u_{2}, \mathscr{\%} /\right\rangle, \mathrm{L},\left\langle u_{n}, \mathscr{O}_{n}\right\rangle\right\rangle\right)$

Theorem 2 . (Idempotency) If $d_{j} \sigma=d$ for all $j$, then

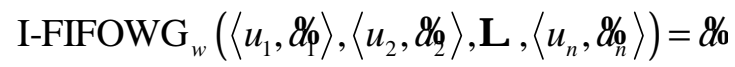

Proof. Since $d_{j} \sigma=\&$ for all $j$, we have

I-FIFOWG $_{\mathrm{w}}\left(\left\langle u_{1}, 8 \hat{\phi},\left\langle u_{2}, \& \&_{2}\right\rangle, \mathrm{L},\left\langle u_{n}, 8 \&_{n}\right\rangle\right)\right.$

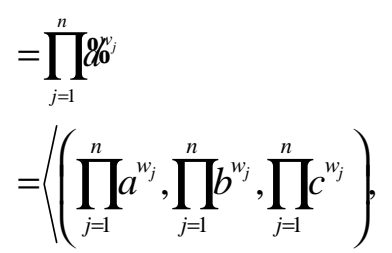

$$
\begin{aligned}
& \left(1-\prod_{j=1}^{n}(1-l)^{w_{j}}, 1-\prod_{j=1}^{n}(1-m)^{w_{j}}, 1-\prod_{j=1}^{n}(1-p)^{w_{j}}\right) \\
& \langle(a, b, c),(l, m, p)\rangle=\& 0
\end{aligned}
$$

Theorem 3. (Monotonicity) If $\mathscr{Q} / \mathrm{j} \leq \mathscr{Q} / \mathrm{c}$ for all $\mathrm{j}$, then

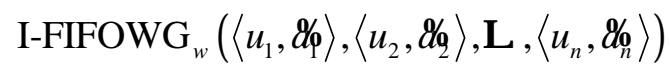

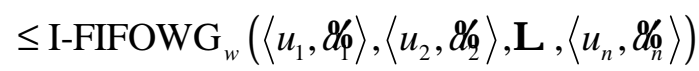

Proof. Let

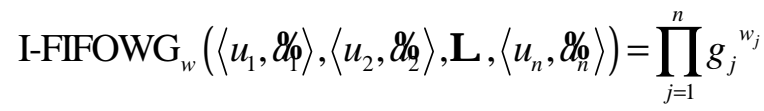

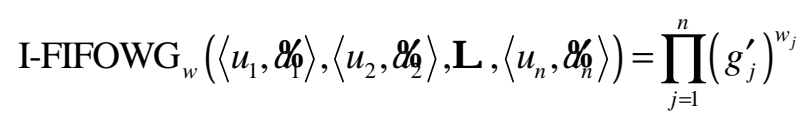

Since $\mathscr{V}_{j} \leq \mathscr{d} / \mathrm{c}$ for all j, it follows that $g_{j} \leq g_{j}^{\prime}$, then 
$\left.\mathrm{I}-F I F O W G_{\mathrm{w}}\left(\left\langle u_{1}, \mathscr{Q} / \mathrm{O}\right\rangle,\left\langle u_{2}, \mathscr{Q} 2 \mathrm{O}\right\rangle, \mathrm{L},\left\langle u_{n}, \mathscr{Q}_{n}\right\rangle\right\rangle\right) \leq$

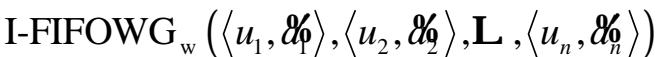

Example 3. Assume we have four FIFOWG pairs $\left.\left\langle u_{j}, \mathscr{O}\right\rangle\right\rangle$ given

$\left\langle u_{1}, \mathscr{d}_{1} \mathrm{p}=\langle 0.4,\langle(0.4,0.4,0.4),(0.1,0.2,0.3)\rangle\rangle\right.$,

$\left\langle u_{2}, q_{2}\right\rangle=\langle 0.2,\langle(0.2,0.3,0.3),(0.3,0.4,0.5)\rangle\rangle$,

$\left\langle u_{3}, \&_{3} 0\right\rangle=\langle 0.8,\langle(0.6,0.6,0.7),(0.1,0.1,0.1)\rangle\rangle$,

$\left\langle u_{3}, \mathscr{Q}_{3} 0\right\rangle=\langle 0.3,\langle(0.6,0.6,0.6),(0.1,0.1,0.1)\rangle\rangle$

That we desire to aggregate using the weighting vector $w=(0.2,0.4,0.1,0.3)$. Performing the ordering the FIFOWG pairs with respect to the first component, we get

$\left\langle u_{\sigma(1)}, \mathscr{Q}_{\sigma(1)}\right\rangle=\langle 0.8,\langle(0.6,0.6,0.7),(0.1,0.1,0.1)\rangle\rangle$

$\left\langle u_{\sigma(2)}, \mathscr{\sigma}_{\sigma(2)}\right\rangle=\langle 0.4,\langle(0.4,0.4,0.4),(0.1,0.2,0.3)\rangle\rangle$

$\left\langle u_{\sigma(3)}, \mathscr{Q}_{\sigma(3)}\right\rangle=\langle 0.3,\langle(0.6,0.6,0.6),(0.1,0.1,0.1)\rangle\rangle$

$\left\langle u_{\sigma(4)}, \mathscr{O}_{\sigma(4)}\right\rangle=\langle 0.2,\langle(0.2,0.3,0.3),(0.3,0.4,0.5)\rangle\rangle$

This ordering includes the ordered intuitionistic fuzzy arguments

$$
\begin{aligned}
& \mathscr{d}_{\sigma(1)}=\langle(0.6,0.6,0.7),(0.1,0.1,0.1)\rangle, \\
& d_{\sigma(2)}=\langle(0.4,0.4,0.4),(0.1,0.2,0.3)\rangle, \\
& d_{\sigma(3)}=\langle(0.6,0.6,0.6),(0.1,0.1,0.1)\rangle, \\
& d_{\sigma(4)}=\langle(0.2,0.3,0.3),(0.3,0.4,0.5)\rangle
\end{aligned}
$$

And from this, we get an aggregated value

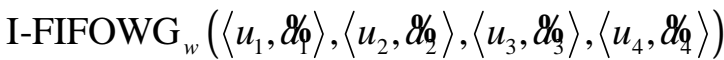

$=\left\langle\left(0.6^{0.2} \times 0.4^{0.4} \times 0.6^{0.1} \times 0.2^{0.3}\right.\right.$,

$0.6^{0.2} \times 0.4^{0.4} \times 0.6^{0.1} \times 0.3^{0.3}$,

$\left.0.7^{0.2} \times 0.4^{0.4} \times 0.6^{0.1} \times 0.3^{0.3}\right)$,

$\left(1-(1-0.1)^{0.2} \times(1-0.1)^{0.4} \times(1-0.1)^{0.1} \times(1-0.3)^{0.3}\right.$,

$1-(1-0.1)^{0.2} \times(1-0.2)^{0.4} \times(1-0.1)^{0.1} \times(1-0.4)^{0.3}$,

$\left.1-(1-0.1)^{0.2} \times(1-0.3)^{0.4} \times(1-0.1)^{0.1} \times(1-0.5)^{0.3}\right)$

$=\langle(0.371,0.437,0.450),(0.814,0.756,0.694)\rangle$
4. Induced fuzzy number intuitionistic fuzzy ordered weighted averaging (I-FIFOWA) operator

Wang [24] propose the fuzzy number intuitionistic fuzzy weighted averaging (FIFWA) operator and fuzzy number intuitionistic fuzzy ordered weighted averaging (FIFOWA) operator.

Definition 10. Let $\mathscr{V}_{j}=\left\langle\left(a_{j}, b_{j}, c_{j}\right),\left(l_{j}, m_{j}, p_{j}\right)\right\rangle$ $(j=1,2, \mathrm{~L}, n)$ be a collection of fuzzy number intuitionistic fuzzy values, and let FIFWA: $Q^{n} \rightarrow Q$, if $F I F W A_{\omega}\left(\mathscr{Q}_{1} Q \mathscr{Q}_{2} \mathrm{gL}, \mathscr{Q}_{n}\right)=\sum_{j=1}^{n} \omega_{j} \mathscr{O}$

$=\left\langle,\left(1-\prod_{j=1}^{n}\left(1-a_{j}\right)^{\omega_{j}}, 1-\prod_{j=1}^{n}\left(1-b_{j}\right)^{\omega_{j}}, 1-\prod_{j=1}^{n}\left(1-c_{j}\right)^{\omega_{j}}\right)\right.$

$\left.\left(\prod_{j=1}^{n} l_{j}{ }^{\omega_{j}}, \prod_{j=1}^{n} m_{j}{ }^{\omega_{j}}, \prod_{j=1}^{n} p_{j}{ }^{\omega_{j}}\right)\right\rangle$

where $\omega=\left(\omega_{1}, \omega_{2}, \mathrm{~L}, \omega_{n}\right)^{T}$ be the weight vector of $\mathscr{Q}_{j}(j=1,2, \mathrm{~L}, n)$, and $\omega_{j}>0, \sum_{j=1}^{n} \omega_{j}=1$, then FIFWA is called the fuzzy number intuitionistic fuzzy weighted averaging (FIFWA) operator [24].

Definition 11. Let $\mathscr{Q}_{j}=\left\langle\left(a_{j}, b_{j}, c_{j}\right),\left(l_{j}, m_{j}, p_{j}\right)\right\rangle$ $(j=1,2, \mathrm{~L}, n)$ be a collection of fuzzy number intuitionistic fuzzy values, An fuzzy number intuitionistic fuzzy ordered weighted averaging (FIFOWA) operator of dimension $n$ is a mapping FIFOWA: $Q^{n} \rightarrow Q$, that has an associated weight vector $w=\left(w_{1}, w_{2}, \mathrm{~L}, w_{n}\right)^{T}$ such that $w_{j}>0$ and $\sum_{j=1}^{n} w_{j}=1$. Furthermore,

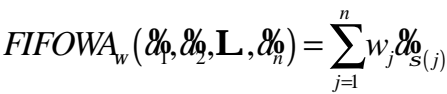

$=\left\langle\left(1-\prod_{j=1}^{n}\left(1-a_{\sigma(j)}\right)^{w_{j}}, 1-\prod_{j=1}^{n}\left(1-b_{\sigma(j)}\right)^{w_{j}}, 1-\prod_{j=1}^{n}\left(1-c_{\sigma(j)}\right)^{w_{j}}\right)\right.$,

$\left.\left(\prod_{j=1}^{n} l_{\sigma(j)}{ }^{w_{j}}, \prod_{j=1}^{n} m_{\sigma(j)}{ }^{w_{j}}, \prod_{j=1}^{n} p_{\sigma(j)}{ }^{w_{j}}\right)\right)$ 
where $(\sigma(1), \sigma(2), \mathrm{L}, \sigma(n))$ is a permutation of $(1,2, \mathrm{~L}, n)$, such that $\mathscr{O}_{\sigma(j-1)} \geq \mathscr{O}_{\sigma(j)}$ for all $j=2$, L , $n$. [24].

In the following, we shall develop an induced fuzzy number intuitionistic fuzzy ordered weighted averaging (I-FIFOWA) operator.

Definition 12. An induced fuzzy number intuitionistic fuzzy ordered weighted averaging (I-FIFOWA) operator is defined as follows:

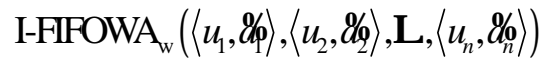

$$
\begin{aligned}
& =\sum_{j=1}^{n} w_{j} \mathscr{V}_{\sigma}(j) \\
& =\left\langle\left(1-\prod_{j=1}^{n}\left(1-a_{\sigma(j)}\right)^{w_{j}}, 1-\prod_{j=1}^{n}\left(1-b_{\sigma(j)}\right)^{w_{j}}, 1-\prod_{j=1}^{n}\left(1-c_{\sigma(j)}\right)^{w_{j}}\right\rangle,\right. \\
& \left.\left(\prod_{j=1}^{n} l_{\sigma(j)}{ }^{w_{j}}, \prod_{j=1}^{n} m_{\sigma(j)}{ }^{w_{j}}, \prod_{j=1}^{n} p_{\sigma(j)}{ }^{w_{j}}\right)\right)
\end{aligned}
$$

where $w=\left(w_{1}, w_{2}, \mathrm{~L}, w_{n}\right)^{T}$ is a weighting vector, such that $w_{j} \in[0,1], \sum_{j=1}^{n} w_{j}=1, j=1,2, \mathrm{~L}, n$, $\mathscr{Q}_{\sigma(j)}=\left\langle\left(a_{\sigma(j)}, b_{\sigma(j)}, c_{\sigma(j)}\right),\left(l_{\sigma(j)}, m_{\sigma(j)}, p_{\sigma(j)}\right)\right\rangle$ is the $\mathscr{Q} \mathbb{C}_{i}$ value of the FIFOWA pair $\left\langle u_{i}, \mathscr{Q}_{i} \mathrm{O}\right\rangle$ having the jth largest $u_{i}\left(u_{i} \in[0,1]\right)$, and $u_{i}$ in $\left\langle u_{i}, Q_{i} 9\right\rangle$ is referred to as the order inducing variable and $\mathscr{Q} /$ c as the fuzzy number intuitionistic fuzzy values.

The I-FIFOWA operator has the following properties similar to those of the IOWA operator [25].

Theorem 4 (Commutativity).

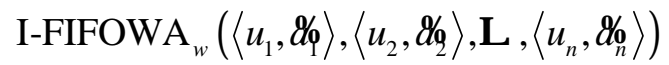

$=\mathrm{I}-F I F O W A_{w}\left(\left\langle u_{1}, \mathscr{O}_{1} \mathrm{O},\left\langle u_{2}, \mathscr{Q}_{2} \mathrm{0}\right\rangle, \mathrm{L},\left\langle u_{n}, \mathscr{Q}_{n} \mathrm{o}\right\rangle\right)\right.$

where $\left(\left\langle u_{1}, \mathscr{O} \%,\left\langle u_{2}, \mathscr{\%} \%, \mathrm{~L},\left\langle u_{n}, \mathscr{C}_{n}\right\rangle\right) \quad\right.\right.$ is any

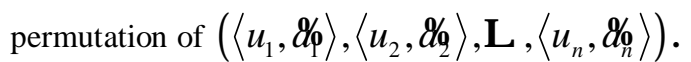

Theorem 4 . (Idempotency) If $d_{j} \sigma=d$ for all $j$, then

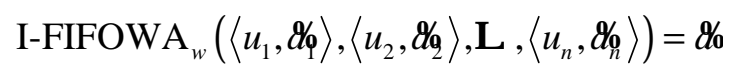

Theorem 6 . (Monotonicity) If $\mathscr{V}_{j} \leq \mathscr{V}_{j}$ c for all j, then

$$
\begin{aligned}
& \left.\left.\operatorname{I-FIFOWA~}_{w}\left(\left\langle u_{1}, \mathscr{Q}_{1}\right\rangle\right),\left\langle u_{2}, \mathscr{Q}_{2}\right\rangle, \mathrm{L},\left\langle u_{n}, \mathscr{Q} /\right\rangle_{n}\right\rangle\right)
\end{aligned}
$$

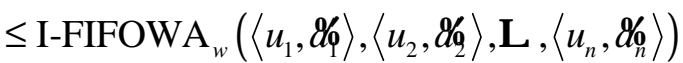

\section{An approach to group decision making with fuzzy number intuitionistic fuzzy information}

In this section, we shall develop an approach to group decision making with fuzzy number intuitionistic fuzzy information as follows.

Let $A=\left\{A_{1}, A_{2}, \mathrm{~L}, A_{m}\right\}$ be a discrete set of alternatives, and $G=\left\{G_{1}, G_{2}, \mathrm{~L}, G_{n}\right\}$ be the set of attributes, $\omega=\left(\omega_{1}, \omega_{2}, \mathrm{~L}, \omega_{n}\right)$ is the weighting vector of the attribute $G_{j}(j=1,2, \mathrm{~L}, n)$, where $\omega_{j} \in[0,1] \quad, \quad \sum_{j=1}^{n} \omega_{j}=1 \quad$ Let $D=\left\{D_{1}, D_{2}, \mathrm{~L}, D_{t}\right\}$ be the set of decision makers, $\mathrm{V}=\left(\mathrm{v}_{1}, \mathrm{v}_{2}, \mathrm{~L}, \mathrm{v}_{n}\right)$ be the weighting vector of decision makers, with $v_{k} \in[0,1], \sum_{k=1}^{t} v_{k}=1$. Suppose that $R_{k} / 0=(p(k))_{m \times n}=\left\langle\left(a_{i j}^{(k)}, b_{i j}^{(k)}, c_{i j}^{(k)}\right),\left(l_{i j}^{(k)}, m_{i j}^{(k)}, p_{i j}^{(k)}\right)\right\rangle_{m \times n}$ is the fuzzy number intuitionistic fuzzy decision matrix, where $\left(a_{i j}^{(k)}, b_{i j}^{(k)}, c_{i j}^{(k)}\right)$ indicates the degree that the alternative $A_{i}$ satisfies the attribute $G_{j}$ given by the decision maker $D_{k},\left(l_{i j}^{(k)}, m_{i j}^{(k)}, p_{i j}^{(k)}\right)$ indicates the degree that the alternative $A_{i}$ doesn't satisfy the attribute $G_{j}$ given by the decision maker $D_{k}$, $\left(a_{i j}^{(k)}, b_{i j}^{(k)}, c_{i j}^{(k)}\right) \subset[0,1],\left(l_{i j}^{(k)}, m_{i j}^{(k)}, p_{i j}^{(k)}\right) \subset[0,1]$, $c_{i j}^{(k)}+p_{i j}^{(k)} \leq 1, i=1,2, \mathrm{~L}, m, j=1,2, \mathrm{~L}, n$, $k=1,2, \mathrm{~L}, t$.

In the following, we apply the I-FIFOWG and FIFWG operator to multiple attribute group decision making with fuzzy number intuitionistic fuzzy information.

Step 1. Utilize the decision information given in matrix $R_{k}^{\circ}$, and the I-FIFOWG operator which has associated weighting vector $w=\left(w_{1}, w_{2}, \mathrm{~L}, w_{n}\right)^{T}$ 


$$
\begin{aligned}
& P / j=\left\langle\left(a_{i j}, b_{i j}, c_{i j}\right),\left(l_{i j}, m_{i j}, p_{i j}\right)\right\rangle
\end{aligned}
$$

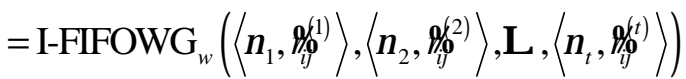$$
i=1,2, \mathrm{~L}, m, j=1,2, \mathrm{~L}, n \text {. }
$$

to aggregate all the decision matrices $R_{k}^{\circ}(k=1,2, \mathrm{~L}, t)$ into a collective decision matrix $R=(r / j)_{m \times n}$, where $\mathrm{v}=\left\{\mathrm{v}_{1}, \mathrm{v}_{2}, \mathrm{~L}, \mathrm{v}_{t}\right\}$ be the weighting vector of decision makers.

Step 2. Utilize the decision information given in matrix $R^{\prime}$, and the FIFWG operator

$$
\begin{aligned}
& P / \sigma\left\langle\left(a_{j}, b_{j}, c_{j}\right),\left(l_{j}, m_{j}, p_{j}\right)\right\rangle \\
& =\mathrm{FIFWG}_{\omega}\left(\% \frac{\%}{\%} \% \mathrm{~L}, \%\right) \\
& i=1,2, \mathrm{~L}, m \text {. }
\end{aligned}
$$

to derive the collective overall preference values $r / \phi i=1,2, \mathrm{~L}, m)$ of the alternative $A_{i}$, where $\omega=\left(\omega_{1}, \omega_{2}, \mathrm{~L}, \omega_{n}\right)^{T}$ is the weighting vector of the attributes.

Step 3. Calculate the scores $S(P / O)(i=1,2, \mathrm{~L}, m)$ of the collective overall fuzzy number intuitionistic fuzzy preference values $P / \phi i=1,2, \mathrm{~L}, m)$ to rank all the alternatives $A_{i}(i=1,2, \mathrm{~L}, m)$ and then to select the best one(s) (if there is no difference between two scores $S(P / \phi)$ and $S(R / \phi)$, then we need to calculate the accuracy degrees $H(r / \phi$ and $H(r / \%)$ of the collective overall fuzzy number intuitionistic fuzzy preference values $P /$ and $\%$, respectively, and then rank the alternatives $A_{i}$ and $A_{j}$ in accordance with the accuracy degrees $H(\% / \phi$ and $H(P / \rho)$.

Step 4. Rank all the alternatives $A_{i}(i=1,2, \mathrm{~L}, m)$ and select the best one(s) in accordance with $S(P / \phi$ and $H(P / \phi(i=1,2, \mathrm{~L}, m)$.

Step 5. End.

\section{Illustrative example}

Let us suppose there is an investment company, which wants to invest a sum of money in the best option [11].
There is a panel with five possible alternatives to invest the money: (1) $A_{1}$ is a car company; (2) $A_{2}$ is a food company;(3) $A_{3}$ is a computer company;(4) $A_{4}$ is an arms company; (5) $\mathrm{A}_{5}$ is a TV company. The investment company must take a decision according to the following four attributes:(1) $G_{1}$ is the risk analysis;(2) $G_{2}$ is the growth analysis; (3) $\mathrm{G}_{3}$ is the social-political impact analysis; (4) $\mathrm{G}_{4}$ is the environmental impact analysis. The five possible alternatives $A(i=1,2, \mathrm{~L}, 5)$ are to be evaluated using the fuzzy number intuitionistic fuzzy numbers by the three decision makers (whose weighting vector $\left.\mathrm{V}=(0.35,0.40,0.25)^{T}\right)$ under the above four attributes (whose weighting vector $\left.\omega=(0.2,0.1,0.3,0.4)^{T}\right)$, and construct, respectively, the decision matrices as listed in the following matrices $R_{k}^{\circ o}=\left(g\left(\gamma_{i j}^{k}\right)\right)_{5 \times 4}(k=1,2,3)$ as follows:

$$
\begin{aligned}
& {[\langle(0.3,0.4,0.5),(0.4,0.5,0.5)\rangle\langle(0.6,0.7,0.8),(0.1,0.1,0.2)\rangle} \\
& \langle(0.4,0.5,0.6),(0.2,0.3,0.4)\rangle\langle(0.5,0.6,0.6),(0.1,0.2,0.3)\rangle \\
& R_{1}=\langle(0.2,0.3,0.4),(0.4,0.5,0.6)\rangle\langle(0.4,0.5,0.6),(0.3,0.3,0.4)\rangle \\
& \langle(0.5,0.6,0.7),(0.1,0.2,0.2)\rangle\langle(0.8,0.8,0.8),(0.2,0.2,0.2)\rangle \\
& \langle(0.7,0.7,0.8),(0.1,0.1,0.2)\rangle\langle(0.5,0.5,0.5),(0.2,0.3,0.4)\rangle \\
& \langle(0.6,0.6,0.7),(0.2,0.2,0.3)\rangle\langle(0.5,0.6,0.7),(0.1,0.2,0.2)\rangle \\
& \langle(0.4,0.5,0.6),(0.2,0.3,0.4)\rangle\langle(0.2,0.3,0.4),(0.4,0.5,0.6)\rangle \\
& \langle(0.7,0.8,0.9),(0.1,0.1,0.1)\rangle\langle(0.1,0.2,0.3),(0.5,0.6,0.7)\rangle \\
& \langle(0.5,0.6,0.6),(0.2,0.3,0.4)\rangle\langle(0.4,0.5,0.6),(0.3,0.4,0.4)\rangle \\
& \langle(0.7,0.7,0.7),(0.1,0.1,0.1)\rangle\langle(0.3,0.4,0.4),(0.4,0.5,0.6)\rangle]
\end{aligned}
$$


Some induced aggregating operators

$$
\begin{aligned}
& {\left[\begin{array}{ll}
\langle(0.2,0.3,0.4),(0.3,0.4,0.4)\rangle & \langle(0.5,0.6,0.7),(0.1,0.1,0.1)\rangle \\
\langle(0.3,0.4,0.5),(0.1,0.2,0.3)\rangle & \langle(0.4,0.5,0.5),(0.1,0.1,0.2)\rangle
\end{array}\right.} \\
& R_{2}^{\prime}=\langle(0.1,0.2,0.3),(0.3,0.4,0.5)\rangle\langle(0.3,0.4,0.5),(0.2,0.2,0.3)\rangle \\
& \langle(0.4,0.5,0.6),(0.1,0.1,0.1)\rangle\langle(0.7,0.7,0.7),(0.1,0.1,0.1)\rangle \\
& \langle(0.6,0.6,0.7),(0.1,0.1,0.1)\rangle\langle(0.4,0.4,0.4),(0.1,0.2,0.3)\rangle \\
& \langle(0.5,0.5,0.6),(0.1,0.1,0.2)\rangle\langle(0.4,0.5,0.6),(0.1,0.1,0.1)\rangle] \\
& \langle(0.3,0.4,0.5),(0.1,0.2,0.3)\rangle\langle(0.1,0.2,0.3),(0.3,0.4,0.5)\rangle \\
& \langle(0.6,0.7,0.8),(0.1,0.1,0.1)\rangle\langle(0.1,0.1,0.2),(0.4,0.5,0.6)\rangle \\
& \langle(0.4,0.5,0.5),(0.1,0.2,0.3)\rangle\langle(0.3,0.4,0.5),(0.2,0.3,0.3)\rangle \\
& \langle(0.6,0.6,0.6),(0.1,0.1,0.1)\rangle\langle(0.2,0.3,0.3),(0.3,0.4,0.5)\rangle]
\end{aligned}
$$

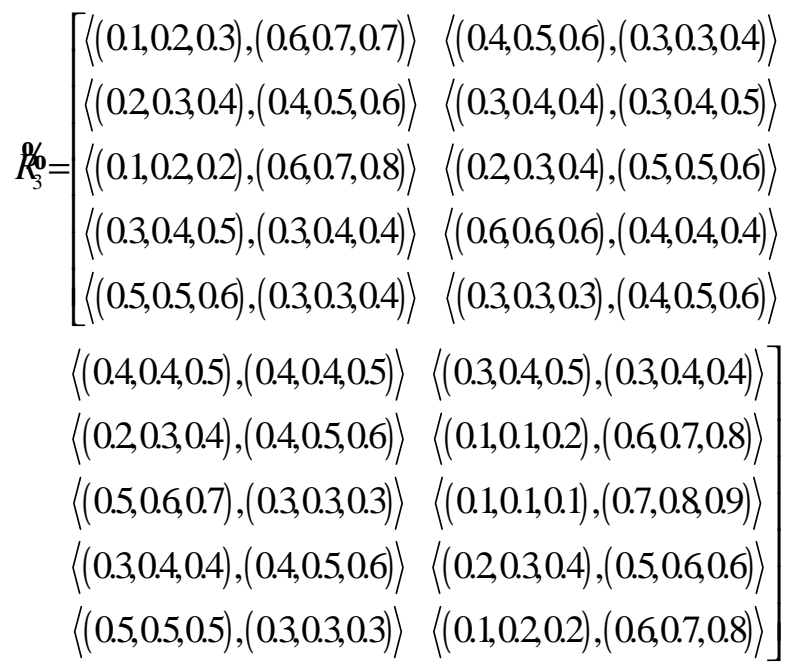

Then, we utilize the approach developed to get the most desirable alternative(s).

Step 1. Utilize the decision information given in matrix $R_{k}^{\prime o}$, and the I-FIFOWG operator which has associated weighting vector $w=(0.20,0.50,0.30)^{T}$, we get a collective decision matrix $R=(\% / 0)_{m \times n}$ as follows:

$$
\begin{aligned}
& {[\langle(0.199,0.307,0.410),(0.452,0.555,0.555)\rangle} \\
& \langle(0.307,0.410,0.512),(0.249,0.350,0.452)\rangle \\
& R=\langle(0.141,0.199,0.307),(0.452,0.555,0.660)\rangle \\
& \langle(0.410,0.512,0.614),(0.165,0.249,0.249)\rangle \\
& \langle(0.614,0.614,0.715),(0.165,0.165,0.249)\rangle \\
& \langle(0.512,0.614,0.715),(0.165,0.165,0.249)\rangle \\
& \langle(0.410,0.512,0.512),(0.165,0.249,0.350)\rangle \\
& \langle(0.307,0.410,0.512),(0.350,0.350,0.452)\rangle \\
& \langle(0.715,0.715,0.715),(0.249,0.249,0.249)\rangle \\
& \langle(0.410,0.410,0.410),(0.249,0.350,0.452)\rangle \\
& \langle(0.512,0.512,0.614),(0.249,0.249,0.350)\rangle \\
& \langle(0.307,0.410,0.512),(0.249,0.350,0.452)\rangle \\
& \langle(0.614,0.715,0.815),(0.165,0.165,0.65)\rangle \\
& \langle(0.410,0.512,0.512),(0.249,0.350,0.452)\rangle \\
& \langle(0.614,0.614,0.614),(0.165,0.165,0.165)\rangle \\
& \langle(0.410,0.512,0.614),(0.165,0.249,0.249)\rangle \\
& \langle(0.141,0.199,0.307),(0.452,0.555,0.660)\rangle \\
& \langle(0.100,0.141,0.199),(0.555,0.660,0.771)\rangle \\
& \langle(0.307,0.410,0.512),(0.350,0.452,0.452)\rangle \\
& \langle(0.199,0.307,0.307),(0.452,0.555,0.660)\rangle]
\end{aligned}
$$

Step 2. Utilize the decision information given in matrix $R^{\prime}$, and the FIFWG operator, we obtain the collective overall preference values $r_{l}$ of the alternatives $A_{i}(i=1,2, \mathrm{~L}, 5)$.

$$
\begin{aligned}
& P / \sigma=\langle(0.388,0.471,0.575),(0.257,0.316,0.352)\rangle \\
& P 2 \%=\langle(0.232,0.314,0.417),(0.331,0.433,0.540)\rangle \\
& \stackrel{8}{3}=\langle(0.207,0.274,0.364),(0.418,0.499,0.602)\rangle \\
& P=\langle(0.386,0.485,0.549),(0.276,0.366,0.398)\rangle \\
& q \%=\langle(0.376,0.447,0.460),(0.302,0.367,0.453)\rangle
\end{aligned}
$$

Step 3. Calculate the scores $S\left(P \gamma_{l}(i=1,2, \mathrm{~L}, 5)\right.$ of the collective overall fuzzy number intuitionistic fuzzy preference values $\% / \phi i=1,2, \mathrm{~L}, 5)$ 


$$
\begin{aligned}
& S(P \not S=0.166, S(P Q \bar{Q})=-0.115 \\
& S(P / \partial)=-0.225, S(R / \%)=0.125 \\
& S(P /)=0.060
\end{aligned}
$$

Step 4. Rank all the alternatives $A_{i}(i=1,2,3,4,5)$ in accordance with the scores $S(P / \phi \quad(i=1,2, \mathrm{~L}, 5)$ of the collective overall fuzzy number intuitionistic fuzzy preference values $8 /(\mathrm{i}=1,2 \mathrm{~L}, 5): A_{1} \mathrm{f} A_{4}$ f $A_{5} \mathrm{f} A_{2} \mathrm{f} A_{3}$, and thus the most desirable alternative is $A_{1}$.

In the following, the I-FIFOWA and FIFWA operators are used to solve the above multiple attribute group decision making problems. We can get the same order of the selection. We find an important feature of the FIFOWG and I-FIFOWA operators is that the argument ordering process is guided by a variable called the order inducing value. Both FIFOWG and IFIFOWA operators essentially aggregate objects, which are pairs, and provide a very general family of aggregation operators. However, we also find difference between two kinds of methods, for the same multiple attribute group decision making problems with fuzzy number intuitionistic fuzzy information, if we emphasize the individual influence, the method based on the I-FIFOWG and FIFWG operators are available; if we emphasize the group's influence, the methods based on the I-FIFOWA and FIFWA operators are available.

Especially, if the triangular fuzzy numbers $\left(a_{j}, b_{j}, c_{j}\right)$ and $\left(l_{j}, m_{j}, p_{j}\right)$ are reduced to the interval numbers $\left[a_{j}, b_{j}\right]$ and $\left[l_{j}, m_{j}\right]$, then, the IFIFOWG or I-FIFOWA operator is reduced to the induced interval-valued intuitionistic fuzzy ordered weighted geometric (I-IIFOWG) operator[40] or induced interval-valued intuitionistic fuzzy ordered weighted averaging(I-IIFOWA); if $a_{j}=b_{j}=c_{j}=\mu_{j}$, $l_{j}=m_{j}=p_{j}=v_{j}$, then the I-FIFOWG or I-FIFOWA operator is reduced to the induced intuitionistic fuzzy ordered weighted geometric (I-IFOWG) operator[40] or induced intuitionistic fuzzy ordered weighted averaging(I-IFOWA) operator[33].

\section{Conclusion}

The traditional induced aggregation operators are generally suitable for aggregating the information taking the form of numerical values, and yet they will fail in dealing with fuzzy number intuitionistic fuzzy information. In this paper, we have developed an induced fuzzy number intuitionistic fuzzy ordered weighted geometric(I-FIFOWG) operator, which take as their argument pairs, called FIFOWG pairs, in which one component is used to induce an ordering over the second components which are fuzzy number intuitionistic fuzzy values and then aggregated. We have studied some desirable properties of the I-FIFOWG operators, such as commutativity, idempotency and monotonicity, and applied the I-FIFOWG operators to group decision making with fuzzy number intuitionistic fuzzy information. Furthermore, we propose the induced fuzzy number intuitionistic fuzzy ordered weighted averaging (I-FIFOWA) operator. Finally an illustrative example has been given to show the developed method.

\section{Acknowledgment}

The author is very grateful to the editor and the anonymous referees for their insightful and constructive comments and suggestions, which have been very helpful in improving the paper. The work was supported by the Humanities and Social Sciences Foundation of Ministry of Education of the People's Republic of China (No.09XJA630010)

\section{References}

[1] K. Atanassov, Intuitionistic fuzzy sets. Fuzzy Sets and Systems 20 (1986) 87-96.

[2] K. Atanassov, More on intuitionistic fuzzy sets. Fuzzy Sets and Systems 33(1989) 37- 46.

[3] L. A. Zadeh, Fuzzy sets. Information and Control 8 (1965) 338- 356.

[4] W. L. Gau and D. J. Buehrer, Vague sets.IEEE Transactions on Systems, Man and Cybernetics 23 (2) (1993) 610-614.

[5] H. Bustine and P. Burillo, Vague sets are intuitionistic fuzzy sets, Fuzzy Sets and Systems 79 (1996) 403-405.

[6] D.F. Li, Multiattribute decision making models and methods using intuitionistic fuzzy sets, Journal of Computer and System Sciences 70 (2005) 73-85.

[7] L. Lin, X.H. Yuan and Z.Q. Xia, Mult icriteria fuzzy decision-making methods based on intuitionistic fuzzy sets, Journal of Computer and System Sciences 73 (2007) 84-88.

[8] Z. S. Xu and R. R. Yager, Some geometric aggregation operators based on intuitionistic fuzzy sets, International Journal of General System 35(4) (2006) 417-433.

[9] Z. S. Xu, Intuitionistic preference relations and their application in group decision making. Information Science 177(11) (2007) 2363-2379. 
[10] Z. S. Xu, Intuitionistic fuzzy aggregation operators. IEEE Transations on Fuzzy Systems 15(6) (2007) 1179-1187.

[11] K. Atanassov and G. Gargov, Interval-valued intuitionistic fuzzy sets, Fuzzy Sets and Systems 31 (1989) 343-349.

[12] K. Atanassov, Operators over interval-valued intuitionistic fuzzy sets, Fuzzy Sets and Systems 64 (2) (1994) 159-174.

[13] J.Q.Wang, Multi-criteria interval intuitionistic fuzzy decision-making approach with incomplete certain information, Control and Decision 21(11) (2006) 1253-1256,1263.

[14] Z. S. Xu, Methods for aggregating interval-valued intuitionistic fuzzy information and their application to decision making, Control and Decision 22(2) (2007) 215-219.

[15] Z. S. Xu and J. Chen, An approach to group decision making based on interval-valued intuitionistic judgment matrices, System EngineerTheory \& Practice 27(4) (2007)126-133.

[16] F. Liu, X.H. Yuan, fuzzy number intuitionistic fuzzy set, Fuzzy Systems and Mathematics 21(1) (2007) 88-91.

[17] X. F. Wang, Fuzzy number intuitionistic fuzzy geometric aggregation operators and their application to decision making, Control and decision 23(6) (2008) 607-612.

[18] Xu Z S, Da Q L. An overview of operators for aggregating information. International Journal of Intelligent Systems 18 (2003)953-969.

[19] F. Herrera, E. Herrera-Viedma, Linguistic decision analysis: steps for solving decision problems under linguistic information, Fuzzy Sets and systems 115(10) (2000) 67-82.

[20] R. R. Yager and D. P. Filev, Induced ordered weighted averaging operators. IEEE Transactions on Systems, Man, and Cybernetics-Part B 29(1999) 141-150.

[21] R. R. Yager and J. Kacprzyk, The ordered weighted averaging operators: Theory and Applications, Kluwer, Boston, 1997.

[22] Z. S. Xu, Uncertain Multiple Attribute Decision Making: Methods and Applications. Beijing: Tsinghua University Press, 2004.

[23] Z. S. Xu, Q. L. Da, An overview of operators for aggregating information. International Journal of Intelligent Systems 18 (2003)953-969.

[24] X. F. Wang, Fuzzy number intuitionistic fuzzy arithmetic aggregation operators, International Journal of Fuzzy Systems 10(2) (2008) 104-111.

[25]Yager R R, Filev D P. Induced ordered weighted averaging operators. IEEE Transactions on Systems, Man, and Cybernetics- Part B 29 (1999) 141-150.
[26] G. W. Wei, Maximizing deviation method for multiple attribute decision making in intuitionistic fuzzy setting, Knowledge-Based Systems 21(8) (2008) 833-836.

[27] Atanassov, K., G. Pasi and R. Yager. Intuitionistic fuzzy interpretations of multi-criteria multi-person and multi-measurement tool decision making. International Journal of Systems Science 36(14) (2005) 859-868.

[28]G.W. Wei, Some geometric aggregation functions and their application to dynamic multiple attribute decision making in intuitionistic fuzzy setting, International Journal of Uncertainty, Fuzziness and Knowledge-Based Systems 2009 17(2) (2009) 179-196.

[29]Z.S.Xu, On generalized induced linguistic aggregation operators, International Journal of General Systems 35(1) (2006) 17-28.

[30] Z. S. Xu, Extended IOWG operator and its use in group decision making based on multiplicative linguistic preference relations, American Journal of Applied Sciences 2(3) (2005) 633-643.

[31] Z.S.Xu, Induced uncertain linguistic OWA operators applied to group decision making, Information fusion, 7 (2006) 231-238.

[32] Z.S.Xu, An approach based on the uncertain LOWG and induced uncertain LOWG operators to group decision making with uncertain multiplicative linguistic preference relations, Decision Support Systems 41(2006) 488-499.

[33]G. W. Wei, Induced intuitionistic fuzzy ordered weighted averaging operator and its application to multiple attribute group decision making. Lecture Notes in Artificial Intelligence 5009(2008)124-131.

[34] Z. S. Xu, Dynamic intuitionistic fuzzy multi-attribute decision making, International Journal of Approximate Reasoning 48(1) (2008) 246-262.

[35] Z. S. Xu, Models for multiple attribute decision-making with intuitionistic fuzzy information, International Journal of Uncertainty, Fuzziness and Knowledge-Based Systems 15(3) (2007) 285-297.

[36] Z. S. Xu, Multi-person multi-attribute decision making models under intuitionistic fuzzy environment, Fuzzy Optimization and Decision Making 6 (3) (2007) 221-236.

[37]G. W. Wei, Uncertain linguistic hybrid geometric mean operator and its Application to group decision making under uncertain linguistic environment, International Journal of Uncertainty, Fuzziness and Knowledge-Based Systems 17(2) (2009)251-267.

[38] T.R. Li, D. Ruan, W. Geert, J. Song, Y. Xu, A rough sets based characteristic relation approach for dynamic attribute generalization in data mining, Knowledge-Based Systems 20(5) (2007) 485-494.

[39] L. Martínez, J. Liu, D. Ruan, J.B. Yang, Dealing with heterogeneous information in engineering evaluation processes, Information Sciences 177(7) (2007)1533-1542.

[40] G. W. Wei, Some induced geometric aggregation operators with intuitionistic fuzzy information and their 
G2W. Wei, X.F. Zhao and R. Lin

application to group decision making, Applied Soft Computing, 10(2) (2010) 423-431.

Published by Atlantis Press Copyright: the authors 